\title{
In silico RNA-seq and experimental analyses reveal the differential expression and splicing of EPDR1 and ZNF518B genes in relation to $K R A S$ mutations in colorectal cancer cells
}

\author{
ÁNGELA L. RIFFO-CAMPOS ${ }^{1-3}$, JOSEFA CASTILLO ${ }^{2}$, AZAHARA VALLET-SÁNCHEZ ${ }^{1,2}$, GUILLERMO AYALA ${ }^{4}$, \\ ANDRÉS CERVANTES ${ }^{2,5}$, GERARDO LÓPEZ-RODAS ${ }^{1,2}$ and LUIS FRANCO ${ }^{1,2}$ \\ ${ }^{1}$ Department of Biochemistry and Molecular Biology, University of Valencia, Valencia; \\ ${ }^{2}$ INCLIVA Institute of Health Research, Valencia, Spain; ${ }^{3}$ Laboratory of Molecular Pathology, \\ Department of Pathology, Faculty of Medicine, Universidad de La Frontera, Temuco, Chile; \\ ${ }^{4}$ Department of Statistics and Operational Research, University of Valencia, Burjassot, Valencia; \\ ${ }^{5}$ Department of Medicine, University of Valencia, Valencia, Spain
}

Received May 3, 2016; Accepted June 11, 2016

DOI: $10.3892 /$ or.2016.5210

\begin{abstract}
Several drugs used for the treatment of colorectal cancer (CRC) are targeted at the epidermal growth factor receptor, but mutations in genes of the $R A S$ family cause resistance to these drugs. Thus, extensive research is being carried out to counterbalance this resistance. The G13D mutation of $K R A S$ is common in humans, and we previously reported that this mutation results in the epigenetic modification of hnRNP proteins, involved in RNA splicing. As aberrant splicing often results in oncogenicity, the present study aimed to identify the genes which show altered splicing patterns in connection with the G13D KRAS mutation. To accomplish this, we first carried out an in silico analysis of RNA-seq databases and found that the distribution of alternative splicing isoforms of genes RPL13, HSP90B1, ENO1, EPDR1 and ZNF518B was altered in human CRC cell lines carrying the G13D KRAS mutation when compared to cell lines carrying wild-type $K R A S$. The in silico results were experimentally validated by quantitative real-time PCR. Expression of the genes EPDRI and ZNF518B was negligible in the $\mathrm{Caco} 2, \mathrm{RKO}$ and SW48 cell lines, which possess wild-type KRAS, while the HCT116, DLD1 and D-Mut1 cell lines, harbouring the G13D mutation, expressed these genes. Moreover, in both genes, the ratio of isoforms was significantly different between the parental DLD1 $\left(^{+}\right.$ G13D) and D-Mut1 cells, in which the wild-type allele had been knocked out. DWT7m cells also expressed both genes. These cells, derived from DLD1, have spontaneously acquired
\end{abstract}

Correspondence to: Professor Luis Franco, INCLIVA Institute of Health Research, Avenida Menéndez Pelayo 4, Accesorio, ES-46010 Valencia, Spain

E-mail: luis.franco@uv.es

Key words: colorectal cancer, anti-EGFR drugs, alternative splicing, RNA-seq analysis a G12D mutation in their single KRAS allele in $20 \%$ of the population. The present data suggest a relationship between $K R A S$ mutations, particularly G13D, and the expression of the $E P D R 1$ and $Z N F 518 B$ genes and expression of their isoforms and provide enhanced understanding of the molecular mechanisms involved in the resistance of CRC cells to anti-EGF receptor therapies.

\section{Introduction}

Colorectal cancer (CRC) is the third most common cancer worldwide, with more than 1.36 million new cases diagnosed annually (1) and, in spite of the existence of targeted therapies, such as those using monoclonal antibodies against the receptor of the epidermal growth factor (2), the long-term survival of patients with advanced metastatic disease remains poor (3). Overexpression of the epidermal growth factor receptor (EGFR) is an aetiological factor in CRC and thus, many efforts have been carried out to counterbalance the effects of EGFR activation. EGFR signalling mainly includes the RAS/MEK/ ERK, PI3K/AKT and PLC $\gamma /$ PKC cascades, but the SRC tyrosine kinases and STAT pathways also participate (4). Members of the RAS family are encoded by three genes, $K R A S, N R A S$ and HRAS. These genes, particularly KRAS, are mutated in many cases of cancer (5) and mutations render the proteins constitutively active (6). Therefore, patients carrying these mutations are not responsive to anti-EGFR drugs. The most frequent KRAS mutations in CRC patients occur in codons 12 and 13 (7), but other mutations also confer sustained activity to the KRAS protein (8).

Clinical data have revealed that many $K R A S$ mutations are heterozygous and that the mutant allele may become dominant when a mutant allele-specific imbalance (MASI) occurs (9). In CRC, MASI is most frequently associated with the G13D mutation $(10,11)$. Altogether, KRAS mutations and MASI contribute to the poor prognosis of metastatic CRC; therefore, it is important to identify a strategy by which to surmount the resistance due to KRAS mutations. 
To accomplish this, it is vital to identify novel potential target genes whose expression depends on KRAS mutations. The availability of human genome sequence data has facilitated the identification of the causes of many monogenic disorders, but the approaches to study complex diseases, such as cancer, often rely on the analysis of transcriptome data, which can be properly carried out by means of RNA-seq analysis (12). This method, which has often been used to analyse the genetic profile of human tumours (see for instance, refs. 13,14), offers the additional advantage of allowing the detection of mRNA isoforms resulting from alternative splicing (15), an event that affects most of the human genes (16).

In a recent study, we showed that the global acetylome of CRC cells depends on KRAS mutational status, several members of the hnRNP family being differentially acetylated in a mutation-dependent manner (8). These ribonucleoproteins are involved in pre-mRNA splicing and export to the cytoplasm (17). Taking into account that aberrant alternative splicing often causes several pathologies, cancer included (18), it is vital to identify the genes whose splicing is altered in connection with KRAS mutation. In this way, various novel therapeutic targets may be found.

We first addressed this question by means of an in silico analysis of RNA-seq databases, and then the presence of mutated $K R A S$-dependent splicing isoforms in the candidate genes was experimentally validated. We report in the present study that two genes, namely ZNF518B and EPDR1, not only exhibit a pattern of isoform abundance dependent on KRAS G13D and G12D mutations, but also that the whole gene expression largely relies on oncogenic KRAS.

\section{Materials and methods}

In silico analysis of RNA-seq data. RNA-seq data were downloaded from the database of Sequence Read Archive (SRA). They corresponded to the human CRC cell lines HCT116 (replicate PJ, SRX378081; replicate PF, SRX378080; replicate, SRX841184), DLD1 (SRX388825), Caco2 (replicate a, SRX209063; replicate b, SRX209064), RKO (ERX183571) and SW48 (ERX183572).

The RNA-seq files were filtered for quality using FASTX-Toolkit (http://hannonlab.cshl.edu/fastx toolkit/). Then, the human genome (version hg19, ftp://ftp. ensembl.org/pub/release-62/) was indexed using Bowtie2 (http://bowtie-bio.sourceforge.net/index.shtml) and the filtered reads were mapped using TopHat v2.0.9 (http://ccb. jhu.edu/software/tophat/index.shtml). The resulting BAM file was converted to SAM format using Samtools v0.1.19 (19) and the Integrative Genomics Viewer (http://www.broadinstitute.org/igv) was used to visualize the variations in the sequences. Once the respective mutations in the oncogenes were validated, the BAM file was processed with the Cufflinks program v2.1.1 (20). The statistical analysis was performed with the CummeRbund package (http://compbio.mit.edu/ cummeRbund/), and for the alternative splicing analysis the spliceR package (21) was used. The resulting lists of genes and isoforms were filtered in function of the KRAS G13D mutation.

Cell culture. The human CRC cell line HCT116 (ATCC CCL-247), DLD1 (ATCC CCL-221) and its isogenic deriva- tive D-Mut1 (a gift from Dr B. Vogelstein) were grown in McCoy's 5A medium (Sigma, St. Louis, MO, USA). The cell lines RKO (Horizon Discovery, Cambridge, UK) and Caco-2 (ATCC HTB-37) were grown in Dulbecco's modified Eagle's medium (DMEM) and the cell line SW48 (Horizon), was grown in RPMI-1640 medium (Sigma). Cells further referred to as DWT7m were derived from the DLD1 cell line. Following knockout of the G13D KRAS allele, the cells acquired a spontaneous KRAS G12D mutation in $20 \%$ of the cell population, as validated by sequencing in our laboratory (see below); they were grown in the same medium as that used for the parental DLD1 cells. All media were supplemented with $10 \%$ heat inactivated foetal bovine serum, $1 \%$ penicillin-streptomycin and $1 \%$ L-glutamine (Sigma), and the cultures were carried out at $37^{\circ} \mathrm{C}$ in a humidified atmosphere containing $5 \% \mathrm{CO}_{2}$. Table I shows the relevant genotypes and sources of the cell lines.

RNA isolation and RT-qPCR analysis. Total RNA was isolated using the TRIzol method (22). RNA (1 $\mu \mathrm{g})$ was treated with DNase I [amplification grade (Invitrogen, Carlsbad, CA, USA)] for $20 \mathrm{~min}$ at $37^{\circ} \mathrm{C}$ in a final volume of $10 \mu 1$. The reaction was stopped with $1 \mu \mathrm{l}$ of $25 \mathrm{mM}$ EDTA and incubation was carried out for $10 \mathrm{~min}$ at $65^{\circ} \mathrm{C}$ and $1 \mathrm{~min}$ at $90^{\circ} \mathrm{C}$. RNA was then retrotranscribed with $\mathrm{M}-\mathrm{MLV}$ reverse transcriptase (Invitrogen) following the manufacturer's instructions.

The cDNA was amplified in a 7900HT Fast Real-Time PCR System (Applied Biosystems, Foster City, CA, USA) using SYBR-Green (Bio-Rad, Hercules, CA, USA) following the manufacturer's instructions. Specific primers for each gene and isoform were used (Table II). Results were analysed by CFX Manager 2.1 software (Bio-Rad) and the relative gene expression was calculated according to the $\Delta \Delta \mathrm{Cq}$ comparative method (23).

Quantitative values are expressed as mean \pm SD. Data in the different qRT-PCR determinations were compared by two-tailed t-test.

\section{Results}

In silico search for genes and isoforms differentially expressed depending on KRAS G13D mutation. SW48 cells, which harbour wild-type KRAS, BRAF, PIK3CA and TP53 (Table I), were used as the control in the RNA-seq comparisons with the other cell lines. It was verified at this stage that the cDNA sequences actually contained the mutations reported in Table I. The reads showed a mutation coverage ranking between $40-60 \%$ for the mutated allele. It was also validated that neither the control SW48 cells nor the RKO and Caco2 cells harboured mutation in codon 13 . The presence of the other mutations reported in Table I for the different cell lines was also verified.

The efficiency of overall mapping was $73.1 \%$ for the data set of the SW48 cell line, $90.9 \%$ for RKO cells, $73.3 \%$ for Caco 2 (average of the two sets) and an average of $89 \%$ was obtained for the three sets of the HCT116 cell line. The reads from DLD1 were poorly aligned, hovering around $70 \%$. In summary, $>70 \%$ of the reads from the total series of data sets were effectively aligned.

The RNA-seq analysis was carried out as described in Materials and methods. To accomplish this, we first compared 
Table I. Commercial cell lines used in the present study.

\begin{tabular}{|c|c|c|c|c|c|}
\hline \multirow[b]{2}{*}{ Cell line } & \multicolumn{4}{|c|}{ Relevant genotype } & \multirow[b]{2}{*}{ Source } \\
\hline & $K R A S$ & $B R A F$ & PIK3CA & TP53 & \\
\hline DLD1 & G13D & wt & E545K; D549N & $\mathrm{S} 241 \mathrm{~F}$ & ATCC CCL-221 \\
\hline HCT116 & G13D & wt & H1047R & wt & ATCC CCL-247 \\
\hline RKO & wt & V600E & H1047R & wt & Horizon Discovery \\
\hline SW48 & wt & wt & wt & wt & Horizon Discovery \\
\hline $\mathrm{Caco} 2$ & wt & wt & wt & E204X & ATCC НTB-37 \\
\hline
\end{tabular}

wt, wild-type.

Table II. Primers used for PCR.

Primers

\begin{tabular}{|c|c|c|c|}
\hline \multirow{2}{*}{$\begin{array}{l}\text { Gene/ } \\
\text { isoform }\end{array}$} & & \multirow{2}{*}{$\begin{array}{c}\text { Amplic } \\
\text { size }\end{array}$} \\
\hline & Forward & Reverse & \\
\hline \multicolumn{4}{|l|}{ ENO1 } \\
\hline 2 & GACTTAGCCGGAGCAGGATG & GAGAGCATTTCAGGGGCCAT & 100 \\
\hline $1+2$ & CTGTTGGCTACACAGACCCC & GCGCTAACTAGCAGGGACC & 90 \\
\hline \multicolumn{4}{|l|}{ HSP90B1 } \\
\hline 1 & CGGGAAGTGGGGGTGAAAAG & GGTTCGGATCCTCACACCTC & 83 \\
\hline \multicolumn{4}{|l|}{ EPDRl } \\
\hline Whole & TGAAACCTGGATTGGCATCTATAC & TGTAGTTTATGGTAAAGGTTTCCTG & 71 \\
\hline 1 & GAGAGGAAGGCGCTGATCC & TGGCTTGGTCAATCTGAAACA & 88 \\
\hline $2 \mathrm{a}$ & GAGGAGGGTCTCTTGGGGAT & GCTGGGTGTTACTGAGTCCC & 96 \\
\hline $2 \mathrm{~b}$ & CGAGGCGGTGGCAGATTATT & TGCTTGGTGGCTTGGTCAAT & 80 \\
\hline 3 & GACATGTGGCCCATCTCTGTAG & ACAATGTTGTCAGCTTCTGCCT & 70 \\
\hline 4 & TCTCCTACGACGGGCTCA & AGGTTTCATCTCTTGCAGGG & 79 \\
\hline \multicolumn{4}{|l|}{ ZNF518B } \\
\hline $1+4+5+6$ & GGGCCTGAGGTTGTGAAACT & AAAACCGTGGCAAGTCCCAT & 91 \\
\hline 1 & GCTACAGGCAGGAATGTTACC & CGCAGTAGGTGCATGATCCC & 75 \\
\hline $2 \mathrm{a}$ & CGGTGTAGACGCCCCTTC & CAGGTAACACCGGCAGGC & 60 \\
\hline $2 b$ & CTGCCGGTGTTACCTGGAAT & GCGCAGCTACTTCTTGGGT & 118 \\
\hline 4 & AGTTTCGAGGTCATTATTCTCTACT & CTGCAGGAGACAGCCTGATT & 83 \\
\hline $5+6$ & GCACAGCAGTGTCAAGTCAA & TAGTCGGCAGGAAGTGAGGG & 78 \\
\hline 6 & CTGGCGTCGTGGCAAGTG & CGTGGACTGCCATGAGTTTC & 116 \\
\hline$A C T B$ & GTGCTATCCCTGTACGCCTC & GAGGGCATACCCCTCGTAGA & 99 \\
\hline
\end{tabular}

the RNA-seq data from the HCT116 and DLD1 cell lines with that of SW48 cells to find the list of isoforms differentially present in the cell lines harbouring the KRAS G13D mutation. Then, the analysis pipeline depicted in the scheme of Fig. 1 was followed. First, the isoform lists from the DLD1 cells and the three sets ( $\mathrm{r} 1, \mathrm{r} 2$ and $\mathrm{r} 3$ ) for the HCT116 cells were compared. With the isoforms coinciding in all of the comparisons, a list of isoforms common to the cells with mutated KRAS and absent from SW48 was constructed. Comparisons were then sequentially made with the lists from the other cell lines that are not mutated in $K R A S$ codon 13, namely RKO and the two duplicates of Caco2 to build lists 1 and 2, and finally, to generate a final list of isoforms, which contained those whose expression was found to be related to the KRAS G13D mutation. These isoforms (Table II) correspond to the ENO1, RPL13, ZNF518B, EPDR1 and HSP90B1 genes. The in silico-determined changes in the expression level of these isoforms are also provided in Table II in FPKM units. These results indicate a $K R A S$ mutation-related overexpression of the ZNF518B, HSP9OBI and EPDRI isoforms and a downregulation of the isoforms of the remaining two genes.

Strategy for RT-qPCR analysis. To validate the results obtained in the in silico analysis, we carried out an RT-qPCR 


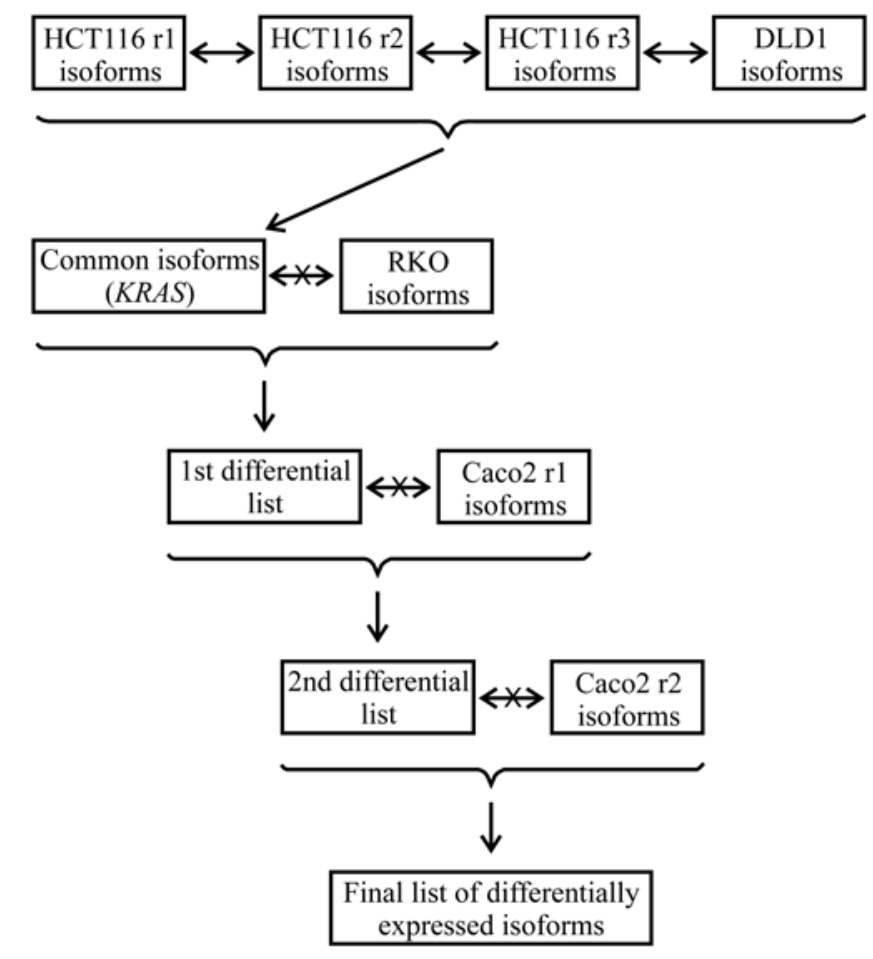

Figure 1. In silico RNA-seq analysis pipeline. The RNA-seq data from the HCT116 and DLD1 cell lines were first compared with that of SW48 cells to construct the list of isoforms differentially present in the cell lines harbouring the KRAS G13D mutation. The isoform lists from the DLD1 cells and the three sets (r1, r2 and r3) for HCT116 were then compared for coincidences, as shown by the double-headed arrows, to yield a list of common isoforms. Comparisons were then sequentially made with the lists from the other cell lines that were not mutated in KRAS codon 13, namely RKO and the two duplicates of $\mathrm{Caco} 2$ to build lists 1 and 2 and, finally, to generate a final list of isoforms, which contains those whose expression depends on the KRAS G13D mutation.

experiment with oligonucleotides amplifying every single isoform of the five genes. First, their exon organization and the composition of the coded transcripts were retrieved from the Ensembl database information (release 84, March 2016, http://www.ensembl.org/index.html) and checked with the manual annotations of the Vega Genome Browser (release 64, February 2016, http://vega.sanger.ac.uk/index.html). To aid the interpretation of the following results, a scheme of the exon organization of the five genes is provided in Fig. 2.

RPL13 has a complex exon organization that gives rise to 15 different isoforms, resulting from multiple exon skipping, intron retention, alternative initiation and termination events. In view of the structure of the gene and of its isoforms (Fig. 2), it was not possible to design primers to specifically amplify isoform 5, differentially expressed in connection with KRAS mutation (Table II). Moreover, this transcript is not translated to protein, and in view of these circumstances, the experimental analysis of RPL13 was not carried out.

In the case of genes ENO1, ZNF518B and EPDR1, both the global expression of the gene and the level of some of their individual isoforms were experimentally analysed by RT-qPCR. To do this, the following strategies were followed. The ENOI isoform 1, reported by spliceR analysis (Table II), cannot be directly amplified. It can be amplified along with isoform 2 , using primers from exon 14. Isoform 2 can be indi- vidually amplified with primers from the region of the intron between exons 4 and 5, which is retained exclusively in this isoform. The value for the level of isoform 1 was then obtained by subtracting the experimentally obtained value for isoform 2 from the value obtained for both isoforms 1 and 2. Primers from exon 3 can also be designed to amplify together all the ENO1 isoforms, with the exception of isoform 2, and therefore, the expression level for whole ENOI was obtained by adding the individual value of isoform 2 to that of the remaining isoforms.

In the case of the EPDRl gene, it was possible to design primers to separately analyse the four isoforms. Isoform 3 can be amplified using a forward primer from exon 1 and a reverse one from exon 2. To amplify isoform 4 , the forward primer was taken from exon 3, and the reverse primer overlapped exons 3 and 6 . To selectively amplify isoform 2 , two alternative sets of primers were used. One of the pairs was taken from exon 4, which is unique to this isoform, while in the second pair the forward primer overlapped exons 4 and 5, and the reverse primer came from exon 5. Both sets of primers gave similar results. Isoform 1 was also able to be individually amplified with primers from exons 3 and 5. Finally, to amplify the whole set of transcripts a pair of primers from the 5' region of exon 6 was taken.

It was also possible to determine the expression of all the isoforms of ZNF518B. The expression of isoform 1 was individually analysed using a forward primer overlapping the junction of exons 3 and 5 and a reverse primer from the 5 ' region of the latter exon. To analyse isoform 6 , primers from exons 2 and 3 were selected. Primers within the intron between exons 3 and 4, which is retained in isoforms 5 and 6 , were used to experimentally determine both isoforms. Then, by subtracting the value of the latter from that of the sum of isoforms 5 and 6 , the expression of isoform 5 was obtained. The level of isoform 4 was determined using a forward primer overlapping the junction of exons 3 and 4 and a reverse primer from exon 4. The individual PCR analysis of isoform 2 was also carried out with a forward primer within exon 1 and a reverse primer overlapping the $3^{\prime}$ end of this exon and the 5 ' end of exon 5. Alternatively, isoform 2 was measured with a forward primer overlapping exons 1 and 5 and with a reverse primer from exon 5. Both pairs of primers gave similar results in the PCR experiments. Finally, using primers from the 5 ' region of exon 3 , the sum of the expression of the isoforms 1 , 4, 5 and 6 was obtained, and thus the expression of the whole gene was easily obtained by adding the value corresponding to the isoform 2 to that of the other four isoforms.

Due to the organization of gene HSP90B1, it was not possible to obtain a value for the expression of the whole gene, neither was the analysis of every individual isoform. Nevertheless, isoform 1, reported by spliceR analysis (Table II), was individually amplified using a forward primer overlapping the junction of the $3^{\prime}$ end of exon 12 and the $5^{\prime}$ end of exon 13 and a reverse primer from the latter exon.

The primers designed as reported above, are listed in Table III. Their quality was routinely assessed for the presence of a single amplification product.

Experimental validation of the in silico data. Once the appropriate primers were designed, we then proceeded to 

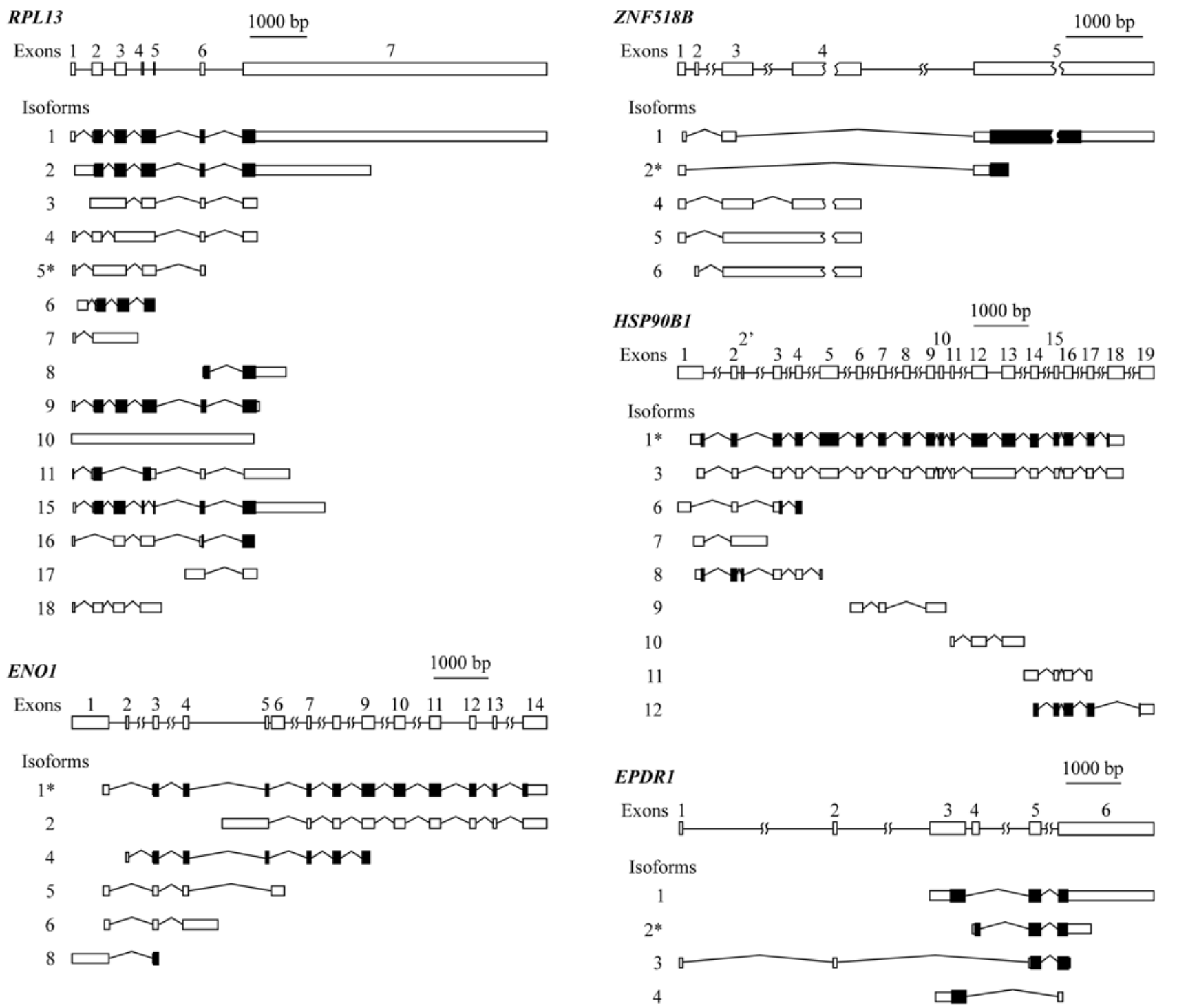

Figure 2. Exon structure of the genes identified by in silico analysis. Exons are represented as boxes and introns as horizontal lines. The structure of the different isoforms reported in the ENSEMBL database is shown below the structure of every gene. Translatable exons are represented as black boxes, while the non-translated exons are depicted as open boxes. The splicing events are represented in the conventional manner. A box wider than the corresponding exon(s) stands for events on intron retention. Putative exons not found in any reported isoform are not included in the scheme. The isoforms reported by the in silico analysis as dependent on KRAS G13D mutation are marked by an asterisk. An apostrophized exon (as in HSP90B1 gene) stands for a non-canonical exon and yet included in some isoform. The bars over the genes mark the size scale of the drawing.

validate the in silico results given in Table II by RT-qPCR. The experimental results for EPDRI and ZNF518B were in good agreement with those of the in silico analysis (Fig. 3). A significant experimental increase was observed in the expression of the isoforms reported by the spliceR analysis when the cell lines carrying the KRAS G13D mutation, for instance HCT116 and DLD1, were compared with lines harbouring wild-type $K R A S$. However, the experimental determination did not corroborate, in general, the results of the in silico analysis in the case of genes ENOI and HSP9OBI and only the increase of the isoform 1 of the latter gene in HCT116 was in accordance with the previous analysis (Table II). Therefore, only the genes EPDR1 and ZNF518B were further experimentally analysed for their global expression and for the expression of their individual isoforms.

To accomplish this, we used the HCT116, DLD1 and D-Mut1 (-/G13D) cell lines, the latter derived from DLD1.
DWT7m cells, which spontaneously acquired a G12D mutation in $20 \%$ of their single KRAS allele, were also used. The Caco2, RKO and SW48 cell lines, which harbour wild-type $K R A S$, were used for comparative purposes. SW48 was the control cell line used in the in silico analysis.

The results obtained with EPDRI showed obvious divergences in the expression of the whole gene among the different cell lines (Fig. 4A). It is noteworthy that the level of mRNA was negligible in the lines harbouring wild-type $K R A S$, while it was significant in HCT116, DLD1 and the cell lines derived from the latter. This result was not anticipated by the in silico analysis. Isoform 2, whose expression was reported by the in silico analysis as being dependent on $K R A S$ G13D mutation, actually was significantly more highly expressed in the two cell lines harbouring that mutation, HCT116 and DLD1. Moreover, its expression level was significantly higher in the D-Mut1 cell line, in which the wild-type KRAS allele was knocked out, than 
Table III. In silico detected isoforms showing differential expression depending on KRAS G13D mutation.

\begin{tabular}{llllllr}
\hline Gene & $\begin{array}{l}\text { Transcript } \\
\text { (TCONS) }\end{array}$ & $\begin{array}{c}\text { ENST id } \\
00000-\end{array}$ & $\begin{array}{l}\text { Diff. } \\
\text { expr. }\end{array}$ & \multicolumn{1}{c}{ Splicing type } & Protein & FPKM \\
\hline ENO1 & 00015435 & 234590 & No & 1 ESI, 1 MESI, 1 A3 & 434 res & -160.8 \\
RPL13 & 00087260 & 491523 & No & 1 ISI, 1 A5SS, 1 ATSS, 1 ATTS & No & -818.7 \\
ZNF518B & 00173221 & 507515 & Yes & 1 MESI & 75 res & +6.8 \\
EPDR1 & 00208723 & 425345 & No & 1 ATSS & 83 res & +9.7 \\
HSP90B1 & 00063866 & 299767 & No & & - & +179.4 \\
\hline
\end{tabular}

The gene and transcript name (TCONS) and their identification in Ensembl database (ENST) are indicated. The column labelled Diff. expr. indicates whether according to the spliceR analysis, the whole gene was differentially expressed. ESI, single exon inclusion; MESI, multiple exon skipping/inclusión; ISI, intron retention/inclusión; A5SS, alternative 5' splicing site; ATSS, alternative transcription start site; ATTS, alternative transcription termination site.
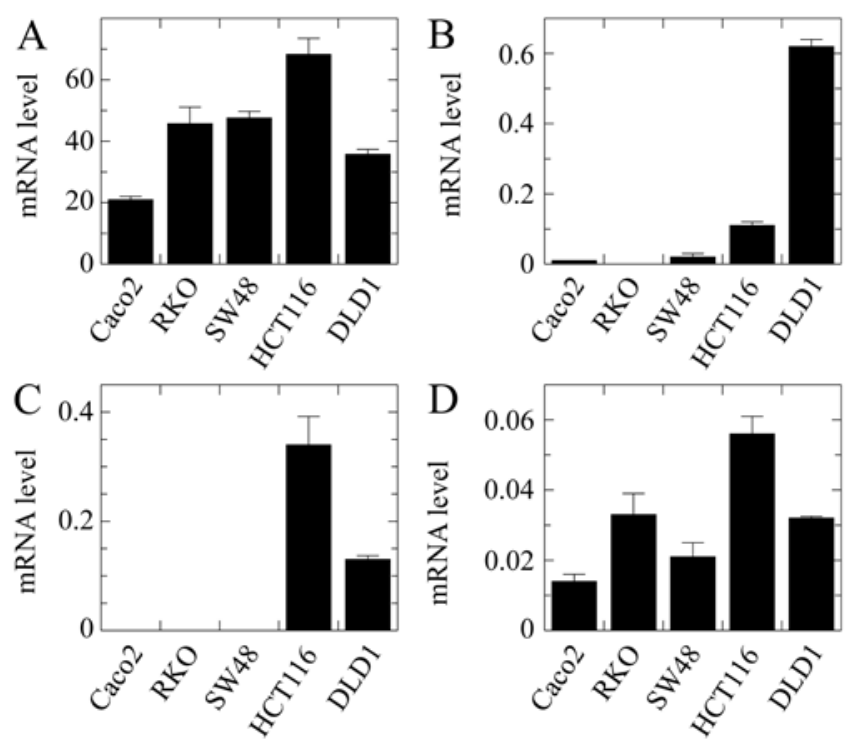

Figure 3. Experimental verification of the expression of the isoforms reported by the in silico analysis. RT-qPCR analyses were carried out in the CRC cell lines used for the in silico studies, with the appropriate primers listed in Table III. (A) Isoform 1 of ENO1. (B) Isoform 2 of EPDR1. (C) Isoform 2 of ZNF518B. (D) Isoform 1 of HSP90B1. The results shown are the average of three determinations \pm SD.

that noted in the parental DLD1 cell line. At this stage of the research we were surprised by the fact that a cell line, initially identified as DWT7, i.e., a DLD1-derived line in which the mutated allele had been knocked out, also expressed the gene and its isoforms. In view of this apparent contradiction, we sequenced the KRAS gene in that line, and found that $20 \%$ of the cells had spontaneously acquired a G12D mutation. These cells were renamed as DWT7m, and the images are lettered accordingly. The expression of isoform 3 was not detected in the cell lines under study.

The global expression of the ZNF518B gene also differed from line to line, being undetectable in the cells with wildtype KRAS (Fig. 5A). In contrast with the results obtained for EPDR1 (Fig. 4), HCT116 cells exhibited a higher expression level than that found in the DLD1 cells. Isoforms 1, 2 and 5 accounted for the majority of the transcripts, isoforms 4 and 6
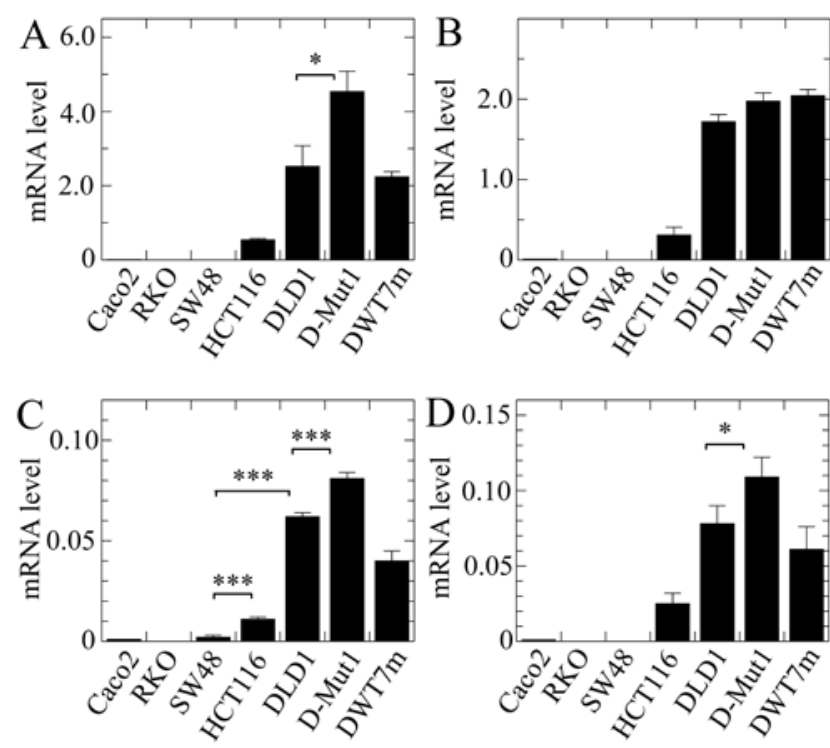

Figure 4. Expression of the EPDRl gene and expression of its isoforms in the CRC cell lines. RT-qPCR determinations were carried out, with the primers provided in Table III, in several CRC cell lines. (A) Whole gene. (B) Isoform 1. (C) Isoform 2. (D) Isoform 4. The levels of mRNA are expressed relative to $\beta$-actin and the average of three determinations \pm SD is shown; ${ }^{* * *} \mathrm{p}<0.001 ;{ }^{* *} \mathrm{p}<0.01 ;{ }^{*} \mathrm{p}<0.05$.

representing only a small minority (Fig. 5). The expression level of the ZNF518B isoforms was also significantly dependent on the presence of either of both KRAS alleles in the DLD1 and D-Mut 1 cells, except in the case of isoform 6, whose extremely low expression did not allow us to draw clear conclusions. The G12D KRAS mutation in the DWT7m cells also resulted in expression of the whole gene and of all its isoforms. Although further discussion on these points is provided later, we wish to emphasize here that the global expression of both genes ZNF518B and EPDR 1 depended on the mutational status of $K R A S$ in the DLD1-based cells.

\section{Discussion}

Since the implementation of high throughput sequencing methods, a plethora of data on transcriptomes virtually 

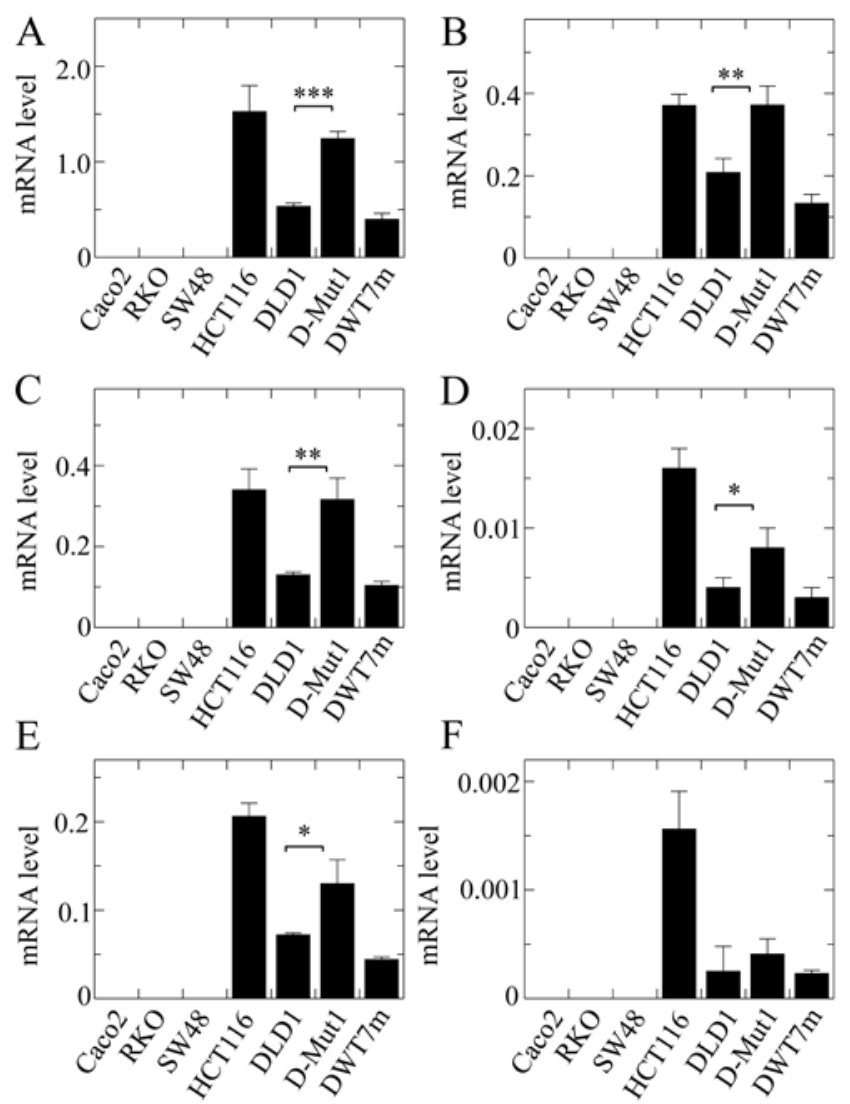

Figure 5. Expression of the ZNF518B gene and expression its isoforms in the CRC cell lines. RT-qPCR determinations were carried out, with the primers provided in Table III, in several CRC cell lines. (A) Whole gene. (B) Isoform 1. (C) Isoform 2. (D) Isoform 4. (E) Isoform 5. (F) Isoform 6 The levels of mRNA are expressed and represented as in Fig. $4{ }^{* * *} \mathrm{p}<0.001$; "** $\mathrm{p}<0.01 ;{ }^{*} \mathrm{p}<0.05$.

covering all of the commonly used cell lines is available. In silico approaches offer the possibility of screening the accessible databases in search of specific transcriptome differences depending on any given variable, using current bioinformatic tools (see for instance, ref. 13). We applied these methods to verify whether the KRAS G13D mutation leads to changes in expression of mRNA isoforms resulting from alternative splicing. The RT-qPCR experiments found that in two out of the five genes reported by the in silico analysis, EPDRI and $Z N F 518 B$, a differential isoform expression occurred when four CRC cell lines carrying the KRAS mutations were compared with three CRC cell lines with wild-type KRAS.

Notably, the global expression of both, EPDRI and ZNF518B genes, and not only the expression of a single isoform, was strongly related to the KRAS G13D mutation. Changes in the expression of these two genes in CRC have been recently reported in relation to the $\mathrm{CpG}$ island methylator phenotype (24). Yet, to the best of our knowledge, this is the first time in which the KRAS G13D mutation-related expression of these genes is reported. Strictly speaking, the differences in the expression of the whole genes and of its isoforms found when comparing Caco2, RKO, SW48, DLD1 and HCT116 cell lines would not allow drawing causal relationships between the KRAS mutational state and the expression of these genes. Nevertheless, in spite of the fact that the above cell lines are not isogenic, the comparison of the behaviour of D-Mut1 and the parental DLD1 does allow us to conclude that the KRAS G13D mutation has an effect on the expression of whole EPDRI and ZNF518B as well as on the relative abundance of their isoforms.

The global expression of both genes was significantly higher in the D-Mut1 than that in the parental DLD1, which behaves as DWT7m (Figs. 4A and 5A). These three cell lines only differ in the mutational status of $K R A S$, since, as previously mentioned, DLD1 is heterozygous carrying a wild-type allele and a G13D mutated one; the wild-type allele is knocked-out in D-Mut1, while DWT7m partially carries allele G12D mutation. The high expression of the genes in D-Mut1 relative to the parental DLD1 cells may seem somewhat surprising, as both cell lines share the same mutated allele. Nevertheless, allelic imbalance in D-Mut1 cells may account for the higher expression of the EPDRI and ZNF518B genes. It has been reported by Soh et al that complete or relative MASI in cancer cells carrying the KRAS mutation results in an enhanced GTPase Ras activity when compared to cells harbouring balanced $K R A S$ mutation. HCT116 cells were included in that study within the balanced group and its GTPase activity was much lower than those of the cells with imbalanced mutations (9). D-Mut1 cells mimic MASI and, therefore, it is tempting to speculate that an increased GTPase activity eventually causes the higher expression of EPDRI and ZNF518B genes.

As mentioned above, not only was the global expression of these genes enhanced in the D-Mut1 cells, but also the expression of their isoforms. The only exceptions were EPDR1 isoform 1 and ZNF518B isoform 6, although the latter result is less reliable due to the low values obtained in the PCR analysis. Notably, the expression of the isoforms reported by the in silico analysis as related to the G13D KRAS mutation, namely EPDR1 isoform 2 and ZNF518B isoform 2, was significantly different between the DLD1 and D-Mut1 cells (Figs. 4 and 5). Moreover, the ratio of ZNF518B isoform 2 to isoform 1 was higher in D-Mut1 (0.85) than that noted in the DLD1 cells (0.63). In addition, of note, the G12D mutation appeared to result in the expression of EPDR1 and ZNF518B, as did G13D. In some way, the oncogenic KRAS is linked to the expression of both genes.

In view of the above comments, it would be worth studying the possible implication of both genes, EPDR1 and ZNF518B, in the oncogenic processes driven by KRAS mutation. EPDRI codes for ependymin, a glycoprotein originally discovered in teleost fishes (for an early review, see ref. 25). A human ependymin homologue was first reported by Nimmrich et al (26), who found that EPDR 1 was highly expressed in CRC cell lines HCT116 and SW480, the latter harbouring a G12V mutation in KRAS. This constitutes an interesting result, since piscine ependymins are extracellular matrix proteins involved in cell adhesion $(27,28)$ and a role in transcriptional control has also been postulated for mammalian ependymin (29). Notably, the sequence containing the topogenic signal for the extracellular location is absent from isoform 2, and this may imply a functional role for the encoded protein.

ZNF518B encodes a zinc-finger protein, which has not yet been isolated. It has been reported that a polymorphism of this gene is associated with gout $(30,31)$. More significant to our purpose is that ZNF518B physically interacts with the 
histone methyltransferase G9a and regulates its activity (32). The deregulation of this enzyme is involved in cancer (33), and therefore, the possible relationships between the KRAS mutation-dependent changes in the expression of the different ZNF518B isoforms and the onset of colorectal malignancies warrant further research. Isoform 2 does not possess the zinc fingers of ZNF518B, but, in view of the results of Maier et al (32), it may be possible that this isoform is still able to interact with G9a to play a role by competing with the whole isoform 1. It is also worth noting that the aberrant alternative splicing of other genes coding for a zinc-finger protein, namely ZNF268, contributes to human malignancies (34). These issues, as well as the possible implications of EPDRI in CRC, are currently being studied in our laboratory.

\section{Acknowledgements}

The present study was supported by grants from the Spanish Ministerio de Economía y Competitividad (FIS PI12/02767 to A.C., FIS PI12/02110 to G.L.-R. and DPI2013-47279-C2-1-R to G.A.), and from Generalitat Valenciana (PROMETEO 2013-005 to A.C. and PROMETEO 2015/006 to G.A.). ALR-C is a fellow of the Grisolia (2012/034) program. We thank Dr B. Vogelstein for the gift of the D-Mutl cell line.

\section{References}

1. Stewart BW and Wild CP (eds): World Cancer Report 2014. IARC Publications, Lyon, 2014.

2. Wong A and Ma BBY: Personalizing therapy for colorectal cancer. Clin Gastroenterol Hepatol 12: 139-144, 2014.

3. Van Cutsem E, Cervantes A, Nordlinger B and Arnold D; ESMO Guidelines Working Group: Metastatic colorectal cancer: ESMO Clinical Practice Guidelines for diagnosis, treatment and followup. Ann Oncol 25 (Suppl 3): iiil-iii9, 2014.

4. Brand TM, Iida M and Wheeler DL: Molecular mechanisms of resistance to the EGFR monoclonal antibody cetuximab. Cancer Biol Ther 11: 777-792, 2011.

5. Prior IA, Lewis PD and Mattos C: A comprehensive survey of Ras mutations in cancer. Cancer Res 72: 2457-2467, 2012.

6. Schubbert S, Shannon K and Bollag G: Hyperactive Ras in developmental disorders and cancer. Nat Rev Cancer 7: 295-308, 2007.

7. Dócs O, Fazakas F, Horváth NL, Tóth L, András C, Horváth Z and Méhes G: Changes of KRAS exon 2 codon 12/13 mutation status in recurrent colorectal cancer. Pathol Oncol Res 21,399-404, 2015.

8. Roda D, Castillo J, Telechea-Fernández M, Gil A, López-Rodas G, Franco L, González-Rodríguez P, Roselló S, Pérez-Fidalgo JA, García-Trevijano ER, et al: EGF-induced acetylation of heterogeneous nuclear ribonucleoproteins is dependent on $K R A S$ mutational status in colorectal cancer cells. PLoS One 10 e0130543, 2015

9. Soh J, Okumura N, Lockwood WW, Yamamoto H, Shigematsu H, Zhang W, Chari R, Shames DS, Tang X, MacAulay C, et al: Oncogene mutations, copy number gains and mutant allele specific imbalance (MASI) frequently occur together in tumor cells. PLoS One 4: e7464, 2009.

10. Hartman DJ, Davison JM, Foxwell TJ, Nikiforova MN and Chiosea SI: Mutant allele-specific imbalance modulates prognostic impact of KRAS mutations in colorectal adenocarcinoma and is associated with worse overall survival. Int $\mathbf{J}$ Cancer 131: 1810-1817, 2012.

11. Malapelle U, Sgariglia R, De Stefano A, Bellevicine C, Vigliar E, de Biase D, Sepe R, Pallante P, Carlomagno C, Tallini G, et al: KRAS mutant allele-specific imbalance (MASI) assessment in routine samples of patients with metastatic colorectal cancer. J Clin Pathol 68: 265-269, 2015.

12. Costa V, Aprile M, Esposito R and Ciccodicola A: RNA-Seq and human complex diseases: Recent accomplishments and future perspectives. Eur J Hum Genet 21: 134-142, 2013.
13. Du J and Zhang L: Integrated analysis of DNA methylation and microRNA regulation of the lung adenocarcinoma transcriptome. Oncol Rep 34: 585-594, 2015.

14. Panagopoulos I, Gorunova L, Zeller B, Tierens A and Heim S: Cryptic FUS-ERG fusion identified by RNA-sequencing in childhood acute myeloid leukemia. Oncol Rep 30: 2587-2592, 2013.

15. Wang W, Qin Z, Feng Z, Wang X and Zhang X: Identifying differentially spliced genes from two groups of RNA-seq samples. Gene 518: 164-170, 2013.

16. Wang ET, Sandberg R, Luo S, Khrebtukova I, Zhang L, Mayr C, Kingsmore SF, Schroth GP and Burge CB: Alternative isoform regulation in human tissue transcriptomes. Nature 456: 470-476, 2008.

17. Matera AG and Wang Z: A day in the life of the spliceosome. Nat Rev Mol Cell Biol 15: 108-121, 2014.

18. Ladomery M: Aberrant alternative splicing is another hallmark of cancer. Int J Cell Biol 2013: 463786, 2013.

19. Li H, Handsaker B, Wysoker A, Fennell T, Ruan J, Homer N, Marth G, Abecasis G and Durbin R; 1000 Genome Project Data Processing Subgroup: The Sequence Alignment/Map format and SAMtools. Bioinformatics 25: 2078-2079, 2009.

20. Trapnell C, Williams BA, Pertea G, Mortazavi A, Kwan G, van Baren MJ, Salzberg SL, Wold BJ and Pachter L: Transcript assembly and quantification by RNA-Seq reveals unannotated transcripts and isoform switching during cell differentiation. Nat Biotechnol 28: 511-515, 2010.

21. Vitting-Seerup K, Porse BT, Sandelin A and Waage J: spliceR: An $R$ package for classification of alternative splicing and prediction of coding potential from RNA-seq data. BMC Bioinformatics 15: $81,2014$.

22. Simms D, Cizdziel P and Chomczynski P: TRIzol: A new reagent for optimal single-step isolation of RNA. Focus 15: 99-102, 1993.

23. Livak KJ and Schmittgen TD: Analysis of relative gene expression data using real-time quantitative PCR and the $2^{-\Delta \Delta C_{\mathrm{T}}}$ method. Methods 25: 402-408, 2001.

24. Slattery ML, Pellatt DF, Mullany LE, Wolff RK and Herrick JS: Gene expression in colon cancer: A focus on tumor site and molecular phenotype. Genes Chromosomes Cancer 54: 527-541, 2015.

25. Shashoua VE: The role of brain extracellular proteins in neuroplasticity and learning. Cell Mol Neurobiol 5: 183-207, 1985.

26. Nimmrich I, Erdmann S, Melchers U, Chtarbova S, Finke U, Hentsch S, Hoffmann I, Oertel M, Hoffmann W and Müller O: The novel ependymin related gene $U C C 1$ is highly expressed in colorectal tumor cells. Cancer Lett 165: 71-79, 2001.

27. Pradel G, Schachner M and Schmidt R: Inhibition of memory consolidation by antibodies against cell adhesion molecules after active avoidance conditioning in zebrafish. J Neurobiol 39: 197-206, 1999

28. Hoffmann W and Schwarz H: Ependymins: Meningeal-derived extracellular matrix proteins at the blood-brain barrier. Int Rev Cytol 165: 121-158, 1996.

29. Shashoua VE, Adams D and Boyer-Boiteau A: CMX-8933, a peptide fragment of the glycoprotein ependymin, promotes activation of AP-1 transcription factor in mouse neuroblastoma and rat cortical cell cultures. Neurosci Lett 312: 103-107, 2001.

30. Jin TB, Ren Y, Shi X, Jiri M, He N, Feng T, Yuan D and Kang L: Genetic variations in the $C L N K$ gene and ZNF518B gene are associated with gout in case-control sample sets. Rheumatol Int 35: 1141-1147, 2015.

31. Zhang XY, Geng TT, Liu LJ, Yuan DY, Feng T, Kang LL, Jin TB and Chen C: SLC2A9 and ZNF518B polymorphisms correlate with gout-related metabolic indices in Chinese Tibetan populations. Genet Mol Res 14: 9915-9921, 2015

32. Maier VK, Feeney CM, Taylor JE, Creech AL, Qiao JW, Szanto A, Das PP, Chevrier N, Cifuentes-Rojas C, Orkin SH, et al: Functional proteomic analysis of repressive histone methyltransferase complexes PRC2 and G9A reveals ZNF518B as a G9A regulator. Mol Cell Proteomics 14: 1435-1446, 2015.

33. Shankar SR, Bahirvani AG, Rao VK, Bharathy N, Ow JR and Taneja R: G9a, a multipotent regulator of gene expression. Epigenetics 8: 16-22, 2013.

34. Zhao Z, Wang D, Zhu C, Shao H, Sun C, Qiu H, Xue L, Xu J, Guo $\mathrm{M}$ and $\mathrm{Li}$ W: Aberrant alternative splicing of human zinc finger gene ZNF268 in human hematological malignancy. Oncol Rep 20: 1243-1248, 2008. 